\title{
Tra bilinguismo e biculturalismo, l'alterità che mi abita
}

Luisa Giacoma

\section{(2) OpenEdition \\ Journals}

Edizione digitale

URL: http://journals.openedition.org/esp/2550

DOI: $10.4000 /$ esp. 2550

ISSN: 2532-0319

Editore

Centre d'Information sur l'Éducation Bilingue et Plurilingue

\section{Edizione cartacea}

Data di pubblicazione: 1 giugno 2018

Paginazione: 89-95

ISSN: 1127-266X

Notizia bibliografica digitale

Luisa Giacoma, «Tra bilinguismo e biculturalismo, l'alterità che mi abita », Éducation et sociétés

plurilingues [Online], 44 | 2018, Messo online il 08 février 2019, consultato il 15 septembre 2020. URL http://journals.openedition.org/esp/2550 


\section{TRA BILINGUISMO E BICULTURALISMO, L'ALTERITÀ GHE MI ABITA}

\section{Luisa Giacoma}

Ma naissance a été annoncée par un mot allemand: Kinderheim. En effet, mon frère et ma scur y furent envoyés afin d'assurer une quinzaine de jours de tranquillité à ma mère et au nouveau-né (moi-même). Comme il était courant chez les familles d'origine allemande comme la nôtre, les enfants pouvaient passer quelques périodes de vacances dans ces structures, qui étaient normalement situées à la mer ou à la montagne. Pour mon frère et ma sour, ce fut la mer. Cela marqua pour eux le début d'une hostilité vis-à-vis de tout ce qui avait trait à l'allemand (y compris la langue), qui les a conduits à se désintéresser complètement de leurs origines.

Mots-clés: italien; allemand; locutions idiomatiques; bilinguisme; biculturalisme.

My birth was announced by a German word: Kinderheim. In fact, my brother and sister were sent away to one, to make sure my mother and the new-born baby (that was me) would have two weeks of quiet. As was usual in families of German descent like mine, children could spend part of their vacations in such a place, usually at the sea or in the mountains. For my brother and sister, it was the sea. For them, it was the beginning of a hostility to everything German (including the language), which led them be totally disinterested by their origins.

Key-zoords: Italian, German, idiomatic expressions, bilingualism, biculturalism

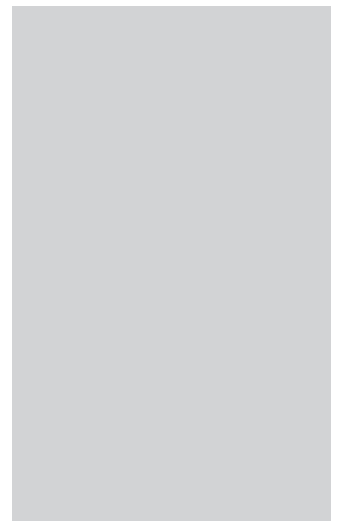

a mia nascita fu preannunciata da una parola tedesca: Kinderheim. Fu lì infatti che vennero portati mio fratello e mia sorella per assicurare un paio di settimane di tranquillità a mia madre e alla nuova nata, che ero io. Come era uso presso le famiglie di origini tedesche come la nostra, i bambini potevano trascorrere alcuni periodi di vacanza in queste strutture, collocate solitamente al mare o in montagna. Ai miei fratelli toccò il mare. Questo segnò per loro l'inizio di un'ostilità nei confronti di tutto quanto fosse tedesco, lingua compresa, che li ha portati a disinteressarsi completamente delle loro origini.

Mia nonna paterna era tedesca e si chiamava Emma, quasi presagissero i suoi genitori la sua futura vita in Italia e volessero evitarle almeno il disagio di sentire il suo nome storpiato, nel 
Tra bilinguismo e biculturalismo, l'alterità che mi abita L. GiACOMA tentativo di adattarlo ai suoni di un'altra lingua. Il suo cognome Mock non lasciava però dubbi sulla sua origine germanica. Dire che fosse tedesca era corretto, ma si trattava di una semplificazione. Nata nel 1897 a Braşov (1), faceva parte di quella popolazione di sassoni che formavano un'isola linguistica e culturale tedesca all'interno dell'impero austro-ungarico. Lasciò la sua terra poco prima che tutto cambiasse per sempre con la prima guerra mondiale e che la Transilvania nella quale era nata fosse ceduta alla Romania. Quando si sposò, venne a vivere nella Valle Sacra, una piccola vallata del Canavese, in Piemonte, della quale era originario mio nonno. Era molto giovane, così il marito, che parlava svariate lingue, acconsentì al suo desiderio di parlare tedesco in casa. Sua sorella Julia la raggiunse dopo pochi mesi e questo certo non facilitò la sua integrazione linguistica nel paese che l'aveva adottata. Mi sembra di sentire ancora quella leggera inflessione tedesca della sua voce, che a volte emerge anche nella mia se non mi sorveglio adeguatamente. Parlava perfettamente l'italiano, ma la fonetica è l'ultima cosa della lingua madre che abbandoniamo quando apprendiamo una nuova lingua. Nel 1915 nacque mio padre e la neomamma trovò naturale parlargli in tedesco e così fece anche con i figli successivi, che impararono l'italiano più tardi con i compagni di giochi e a scuola.

Il mio perenne bilico tra due lingue e due culture è nato così, senza confini netti tra una e l'altra, creandomi talvolta delle false certezze. Un esempio significativo è quello del cucchiaio d'argento, che noi bambini ricevevamo in dono alla nascita. Ho ancora il mio, nel cassetto delle posate, sopravvissuto ai vari traslochi. Nel Nord Europa veniva regalato per augurare prosperità, come testimonia il modo di dire tedesco mit einem silbernen Löffel im Mund geboren sein (lett.: 'essere nato con il cucchiaio d'argento in bocca') e la sua variante più preziosa mit einem goldenen Löffel im Mund geboren sein (lett.:'essere nato con il cucchiaio d'oro in bocca'). In entrambi i casi il significato idiomatico è quello di 'essere nato in una famiglia agiata'. Ora, pur essendo il fatto preso a modello dell'espressione idiomatica tedesca totalmente trasparente e permettendo pertanto una traduzione letterale, il significato italiano è solo quello composizionale mentre nel passaggio da una lingua all'altra viene meno quello idiomatico. Ho scoperto questo fatto molto tardi, quando ho cominciato a collaborare al mio primo dizionario di espressioni idiomatiche (Fenati et alii. 2009). Fino a quel momento non avevo avuto infatti il minimo sospetto che si trattasse di un'espressione 
Tra bilinguismo e biculturalismo, l'alterità che mi abita L. Giacoma idiomatica solo tedesca, perché a casa nostra girava, tradotta in italiano, carica anche di un significato idiomatico che le attribuivamo solo noi. La selezione naturale di amici e conoscenti di ambito tedesco poi, non ci metteva certo in guardia da tali rischi. Con la stessa inconsapevolezza, mio padre diceva ghitarra, avvicinandosi di più al tedesco Gitarre che all'italiano chitarra (la

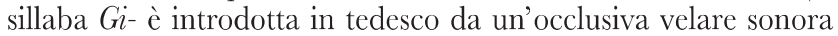
e può quindi essere resa graficamente in italiano con ghi-).

Un altro punto critico per me è la punteggiatura che segue regole diverse nelle due lingue e che mi manca in certi momenti. Il tedesco, ad esempio, mette sempre una virgola prima della proposizione oggettiva introdotta da ich denke, dass... "io penso che...'. Mi irrita non metterla in italiano, è così "pulita" quella separazione, aiuta la lettura.

Naturalmente verrebbe da pensare che, in un ambiente così, l'apprendimento del tedesco sia stato spontaneo e privo di sforzi per me. Questo è indubbiamente vero per la fonetica, ma per quanto riguarda il lessico, la sintassi e tutto il resto ho dovuto impegnarmi non poco anch'io. Mentre sono indubbiamente nata nel biculturalismo, il bilinguismo è stata una lenta e faticosa risalita controcorrente verso le mic origini (ai ricordi dell'infanzia appartengono anche le visite dei parenti che nel frattempo avevano abbandonato l'Ungheria per trasferirsi in Germania, come molti altri sassoni della Transilvania). Ho studiato intensamente tedesco per molti anni, ho vissuto in Germania, sono diventata germanista, ho insegnato all'università, ho lavorato come interprete e traduttrice, ho scritto numerosi dizionari di Tedesco-Italiano, ma ho potuto considerarmi bilingue a tutti gli effetti solo quando ho tenuto i corsi in tedesco per due anni all'Università di Dresda.

Mi sono spesso domandata perché questa inenarrabile fatica non mi sia stata risparmiata dalla mia famiglia, che pure ne avrebbe avuto i mezzi. Mia nonna, mio padre e mia zia erano infatti gelosi di quel codice segreto col quale potevano comunicare tra loro senza essere compresi dagli altri e furono unanimi nel dichiarare che la nuova generazione non avrebbe dovuto imparare quella variante di tedesco, che avrebbe potuto essere di ostacolo per l'apprendimento dell'Hochdeutsch, cioè del tedesco standard. Così io morivo di curiosità quando loro parlavano e mi sforzavo di captare qua e là qualche parola da me conosciuta e da quella intuire qualche brandello di discorso. Ho dovuto però attendere fino all'università per imparare veramente il tedesco, ma a quel punto mia nonna era mancata e con lei si 
Tra bilinguismo e biculturalismo, l'alterità che mi abita L. GiACOMA erano ridotte le possibilità di parlare il tedesco in casa.

$\mathrm{Al}$ contrario dei miei fratelli, ero totalmente affascinata da questa lingua fin da piccola, adoravo sentirlo parlare in casa. Mia nonna cominciò subito a insegnarmi le lettere, i numeri e qualche parolina, ma non andò mai oltre per i motivi appena accennati. Intuendo qualcosa, che i miei studi successivi rivelarono essere la prima e la seconda rotazione consonantica del germanico prima e dell'alto tedesco antico poi, gliene chiedevo insistentemente di nuove, cercando ingenuamente di trovare corrispondenze che permettessero di trovare parole tedesche partendo da quelle italiane. Mia nonna stava al gioco senza spazientirsi, ma su una cosa fu irremovibile: non esisteva alcuna relazione meccanica tra tedesco e italiano che facesse sì che, ad esempio, alla consonante " $t$ " corrispondesse sempre un'altra determinata consonante in italiano. Naturalmente fui piuttosto delusa. Molti anni più tardi, durante il corso di storia della lingua tedesca, scoprii che qualche corrispondenza invece c'era. Senza entrare in questo complesso argomento, che richiederebbe anche un confronto col latino prima e l'italiano poi, desidero solo osservare come il semplice fatto di essere esposti ad un ambiente bilingue porti fin da subito a porsi domande che non emergerebbero in un contesto monolingue.

La cucina era naturalmente il luogo privilegiato di incontro tra le culture di famiglia. Così mia madre, modenese, cercava di carpire alcune ricette, che mia nonna si ostinava a non darle. Le donne all'epoca le custodivano gelosamente come un segreto da non confidare mai a nessuno, ma mia madre non si lasciò scoraggiare: "Che cosa prepara di buono oggi?" domandava. "Gemüse" rispondeva mia nonna senza aggiungere alcuna spiegazione. Mia madre allora osservava i fagioli lessi che venivano scolati e passati in una padella con olio, aglio, farina, alloro e scorza di limone. Qualche giorno più tardi stessa scena, ma con le lenticchie, poi con le patate, con i fagiolini... La risposta era sempre un laconico "Gemüse". Mia madre correva ad annotare in un'altra stanza le ricette chiamandole Gemüse di fagioli, Gemüse di lenticchie, Gemüse di patate... I miei fratelli penso che credano tutt'ora che Gemüse sia un modo di cuocere le verdure in umido e non semplicemente l'equivalente tedesco di 'verdure'! In questa sorta di prestito casalingo dal tedesco poco importava ovviamente che il prefisso Ge- rivelasse la presenza di un nome collettivo... Maggiore fortuna hanno avuto i Knödel (una sorta di grande gnocco a forma di pallina con al centro un'albicocca o una prugna. Dopo la lessatura viene passato nel pangrattato 
Tra bilinguismo e biculturalismo, l'alterità che mi abita L. GiACOMA abbrustolito e servito con lo zucchero), i Krapfen e le Palatschinken (si tratta di una crêpe dolce, ripiena di marmellata e ricoperta di zucchero) che entrarono a far parte del lessico familiare senza slittamenti semantici, ma solo con qualche adattamento fonetico e ortografico: knodel, crafen e palacinte.

Ma le influenze di area tedesca non finivano qui. Avevamo come vicina di casa un'anziana signora austriaca che, rimasta vedova, tirava avanti affittando le stanze del suo grande appartamento a studenti universitari. Sapeva preparare torte meravigliose che allora si potevano mangiare solo in Austria o da lei, se si aveva la fortuna di essere invitati quando le sfornava. Ad ogni nostro compleanno arrivava con una delle sue torte profumate di cioccolato, nocciole o frutta deliziosa e questo era per noi il regalo più atteso. Negli anni diventò amica di mia madre e cominciò a insegnarle una ad una le sue ricette, con il ritmo di circa una all'anno. Aveva tenuto segreta solo l'ultima, perché se avesse rivelato anche quella, cosa mi avrebbe potuto regalare per il mio compleanno? Poco dopo la signora Bertocchi (questo era il suo nome) morì e per tanti anni provai a imitare la sua ultima ricetta, ma nessuna era come l'originale. Poi è arrivato internet e una sconosciuta signora austriaca ha condiviso con me la sua ricetta e così ho ritrovato i sapori e i profumi della torta di ribes della mia vicina di casa.

La cucina non era l'unico aspetto della nostra vita influenzato dalla cultura tedesca. A Pasqua i miei fratelli ed io avevamo una gioia in più rispetto ai coetanei: l'Osterhase (2) infatti aveva lasciato per noi le uova di cioccolata nascoste tra le piante e i fiori del giardino e noi ci precipitavamo fuori a raccoglierle, facendo a gara a chi ne trovava di più. Tornavamo poi a casa col bottino nelle tasche e nel bordo della maglia tirato su a mo' di cestino improvvisato. Si trattava di uova piccole come cioccolatini, molto diverse da quelle tipicamente italiane, grandi e con la sorpresa dentro. Gli amici dei nostri genitori, nel timore che ci sentissimo emarginati rispetto ai nostri amichetti, erano soliti però regalarci anche un uovo grande con sorpresa. In questo avere l'una e l'altra cosa io vedo una metafora perfetta della ricchezza di chi è bilingue.

I miei compagni di scuola mi consideravano tedesca a tutti gli effetti, non tanto per ascendenza famigliare, quanto, credo, per l'educazione e per il modo di parlare. Facevo, ad esempio, un uso inconsapevolmente eccessivo dell'imperativo, modo assai più frequente in tedesco che in italiano. Questo irritava naturalmente i miei compagni, abituati a modi meno imperiosi. Io con 
Tra bilinguismo e biculturalismo, l'alterità che mi abita L. Giacoma l'imperativo ci ero nata, non mi accorgevo quando era di troppo. "Vuoi sempre comandare" mi dicevano e io mi stupivo di quell' affermazione. Con ogni evidenza il tedesco, che è la lingua del comando per antonomasia, non veniva sufficientemente addomesticato dal passaggio attraverso l'italiano, ma anzi in qualche misura lo connotava senza peraltro che io ne avessi consapevolezza.

A scuola le mie origini venivano a volte tirate in ballo a proposito del nazismo. Ricordo una mia compagna delle elementari che, mentre la maestra spiegava la tragedia dei campi di concentramento, si voltò verso di me scandalizzata e con l'aria di dirmi: "Ma cosa hai fatto!". Difficile spiegare che in quegli anni i miei parenti erano ancora in Ungheria, molto lontani dagli orrori nei quali mi vedevano coinvolta per il solo fatto di parlare tedesco! Anzi fu proprio questa lingua a salvare la vita a un giovane durante i rastrellamenti che non risparmiarono neppure casa nostra. Mia nonna lo aveva nascosto in un frigo staccato in cantina, ma fu il fatto di parlare perfettamente la loro lingua a convincere $\mathrm{i}$ soldati tedeschi a non perquisire la casa in modo troppo capillare. Così passarono oltre al frigo senza aprirlo e il ragazzo si salvò.

Nel 2015 e 2016 ho vissuto a Dresda, grazie ad una fellowship presso la Technische Universität, una delle undici università di eccellenza della Germania. Non so come descrivere la sensazione di essere tornata a casa, provata vivendo in Sassonia, più che in altre parti della Germania dove avevo vissuto prima. Tante piccole cose che mi facevano sentire diversa in Italia, lì erano largamente condivise. Mi sentivo finalmente una persona "normale" e avevo dato casa a una parte di me. Quell'esperienza è stata una rivelazione fondamentale: una parte di me non è solo tedesca, è sassone.

Essere a metà tra due lingue e due culture è come essere seduti a cavalcioni sul crinale di una montagna che segna il confine tra due stati. Si è a metà di qua e a metà di là, ma in nessun luogo interi, se non in quell'unico punto di incontro. Si tratta di un osservatorio privilegiato perché le due realtà appaiono in una visione d'insieme che non si potrebbe avere stando seduti altrove. Una parte di noi sarà sempre un po' estranea all'altra, come se due gemelli vivessero in una sola persona. Questo stato di cose ha influenzato anche tutta la mia vita lavorativa, nella quale ho sempre cercato di non sbilanciarmi di qua o di là per mantenere la mia interezza. Ho scritto numerosi dizionari di Tedesco-Italiano nei quali sono al tempo stesso germanista e ita- 


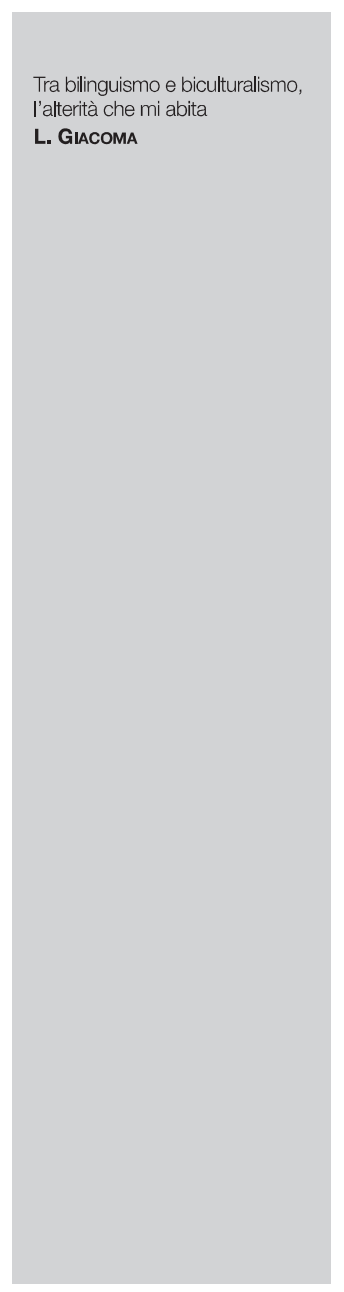

lianista, insegno tedesco in Italia e italiano (in tedesco!) in Germania. Questa doppiezza è stata però a volte un inciampo nel mondo accademico, che mi considerava troppo germanista per gli italianisti e troppo italianista per i germanisti. Io invece vado fiera della mia identità e dell'alterità che con essa vive.

\section{NOTE}

(1) La città è sita nella zona dei Siebenbürgen, nome tedesco della Transilvania che significa letteralmente 'sette borghi', dal numero delle città fondate qui dai tedeschi, tra cui la stessa Braşov.

(2) Letteralmente 'coniglietto di Pasqua'. Nella tradizione tedesca è un coniglietto che il giorno di Pasqua nasconde le uova che porta ai bambini. 